\title{
New Approach For Time Efficent Backup and Restore on on-line Server using Android Platform
}

\author{
PROF. R. C. Dharmik ${ }^{1}$, Sangharsha B. Lanjewar ${ }^{2}$ \\ ${ }^{1}$ (Department of Information Technology Yeshwantrao Chavhan College of Engineering) \\ Nagpur, Maharashtra, India \\ ${ }^{2}$ (Dept. Of Computer Science and Engineering Yeshwantrao Chavhan College of Engineering) \\ Nagpur, Maharashtra, India
}

\begin{abstract}
: while many solutions for making backup and restoring data are known for servers and desktops, mobile devices pose several challenges, mainly due to the differentiating in devices, vendors, operating system and version available in mobile market. We introduce a new backup and restore approach for mobile devices, which helps to reduce the effort in saving and restoring personal data. Our approach is minimize time in efficient way for backup and restoring the data on on-line server using android platform. We are using compression (ZIP) which will reduce the size of file which we are interested to backup and we also use some other component which helps us to reduce the time in backup and efficient storage at the server side. Using of various compression techniques such as RLE, Lempel ziv, wavelets can introduce overhead for maintaining different compression for different purpose. Instead of XML, we are using PHP, the most advantage of using php is that it store file as fragmented manner and did not need any extra effort to do. Also MYsql is far better because it have the flexibility (using SQL commands like SELECT, DELETE, SORT, INSERT, etc.) to easily manipulate the data.
\end{abstract}

Keywords: Backup and restore; mobile devices; protocol; sync;

\section{INTRODUCTION}

B ackup is a crucial task, since hardware faults and software or human errors can lead to the loss of important information. In addition to faults, backups are even more important for devices such as laptops and Smartphone's, since they are more prone to loss or to theft. Currently, Smartphone's are used more as handheld computers than as mobile phones, and consequently a lot of data is stored in those devices. This makes more critical the need to keep data stored on thosel devices safe from losses. In addition, the rapid technological evolution in mobile devices makes it more difficult to restore data saved from old devices to new ones. Thus, mobile devices pose new challenges for the backup and restore problem. Making backups on external memory devices, such as on Secure Digital (SD) cards or on laptop disks, suffers from the same risks of failure or loss. As Smartphone's tend to be always connected to the Internet, it seems natural to move the information online and to provide backup and restore services based on the cloud computing paradigm, which is considered to be more reliable and less expensive by end users. This approach reduces also the risk of data loss and decouples the data from a specific device.

The main goal of this work is to provide backup and restore of mobile device data on on-line server in minimize time (the required time should be less than the original time).Using compression techniques time minimization can be achieved. With such system user can access their data at any time with less waiting time.

\section{STATE OF THE ART}

According to [1], backups can be classified in several types; it is possible to distinguish the data repository model in full backups vs. incremental backups, data can be stored in a file based or a device-based style, and the data repository management can be classified as on-line vs. off-line; those approaches can be combined in different ways, according to accessibility, security and cost needs. In case of failure, a full backup is able to restore the entire content of a device: this process is slow in the backup phase, introduces a huge overhead in the data stored, but allows for faster restores. On the contrary, incremental backups reduce backup times and sizes but imply higher restore times. Backups can operate on files (file-based approach) or on data physically saved on the disk (device-based approach): although a file-based approach: tends to be slower than a device-based bac more flexibility and it is easier to manage. permit to save and restore data while the $s$ while off-line backups require the system backups are more convenient, as they do not users' work, but are more complex to hand needs to deal with updates carried out during cases, backups can be stored locally, e.g. device, or remotely, e.g., on a remote server.

\section{METHODOLOGY}

Developing a system which incorporated the implementation of backup and restore of mobile data on android platform with reduce in time using some compression and encoding method for reducing data size of mobile device and there will be benefits of this encoding and compression method in time required to backup and restore of mobile device data on online server. We are mainly using encoding methods such as [6]Zip compression. [2] Compression uses what is known as an algorithm (any set of instructions to be followed in order) to take a data file as input and make sure the output is a much smaller file. As an example, take a string of characters"cheese and ham and more cheese".

How else could this string of characters be represented? Well, we could make up a code. Notice the words "and" and "cheese" appear twice in the string, we could encode (compress) the string as follows: $\mathrm{b}=$ "cheese" $\mathrm{c}=$ "and" to get: $\mathrm{b}$ $\mathrm{c}$ ham $\mathrm{c}$ more $\mathrm{b}$. 
If this is applied to a whole file only concentrating on frequently used code or text, the file could be made considerably smaller.

\section{For backup and restore different steps are followed:}

\section{A. Creating a data for backup}

The data is created by different genre of information such as contacts, images, sound files, video files, and calendar events.

\section{B. Store data as separate in application}

Each data should be collected separately in application folder of backup and restore.

\section{Line of Action}

The selected data then compress at the client router and then send to the server for backup. Same procedure is performed for restoration. At the restore time server router will compress and then send to the client.

\section{PLAN OF WORK}

1. First, we have to develop an application for android device using Android SDK which requires JDK for selection of multiple data which need to be backup on online server.

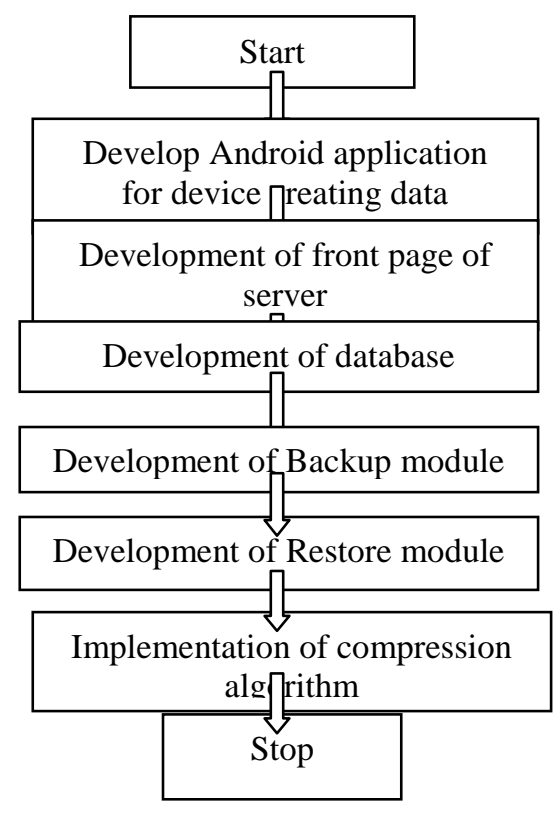

Fig1: Flow of work

2. Second, we want front page of server where we create a user login for an individual user to create their account for storing /backup data of mobile device in his or her personal account.

3. For storage of user mobile data, we need to maintain a record or databases for which, we use mysql.

4. In backup module, we incorporate some backup technique such as full backup or incremental backup system with content provider by android.

5. Similarly for restore, we can implement only selected file should be restore on mobile device or full restoration from server by using fetch call i.e. On restore $\operatorname{method}()$
6. [3]In compression module, we trying to overcome the time required to backup and restore on mobile to server and server to mobile respectively using above define encoding and decoding techniques.

\section{DEVELOPMENT}

There are six modules in the project:

1. Development of application

2. Development of Front page using php software.

3. Development of database using mysql.

4. Backup module.

5. Restore module.

6. Compression on data.

1. Development of application

In this module, application is developed using java eclipse and ADT emulator.

2. Development of Front page using php software In this module, front page developed for efficient segmentations of data using php over $\mathrm{xml}$ as this need more code to write.

3. Development of database using mysql

In this module, mysql will help to create database for efficient storage and operation over SQL Lite.

4. Backup module

In this module, backup of data is performing from mobile phone to on-line server without compression.

5. Restore module

In this module, restore of data is performing from on-line server to mobile device without compression.

6. Compression on data

In this module, compression technique is use for compress data on mobile phone and then tries to backup and restore mobile data using compression.

\section{Development of application.}

In this module, application is developed using java eclipse and ADT.

The following steps are involved in creation of application. First create android project. This android project will create java file with project name. Before starting project code import necessary file from android package. In this case is

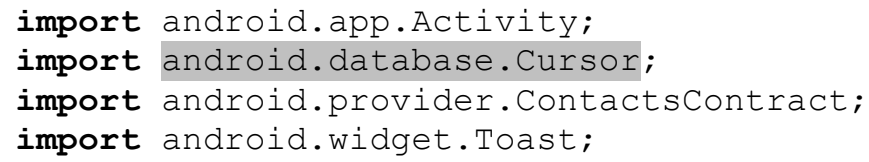

First step: Install android packages. It should be called before creating any contact. We can't run application without initializing android package first. We are storing contact in an array list (Array list <contact>) similarly the other . 
Second step: To read a contact from device we need to get permission. This permission we will get from android manifest.xml. We have to define in manifest as this can be use for other function like message and call $\operatorname{logs}$, the manifest is <uses-permission

android: name="android.permission.READ_CON TACTS" $></$ uses-permission $>$

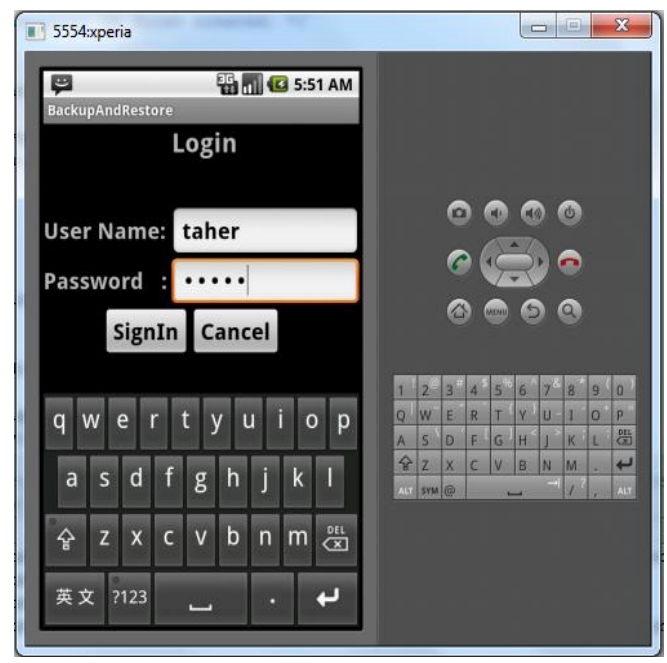

Fig 2: User login window

Here are the steps needed to follow in application:

1. We need to create a contact and to store it for application.

2. Create one contact with its name, phone number by clicking on contact button.

3. Repeat the process from 2 if we want to create more contact.

4. The above process can be repeat for other function (message,call logs) with slightly difference .

5. Then run the application backup and restore for taking up backup and restoration of device data(contacts, message and call logs).

\section{Development of php}

In this module we need to create php page for interaction between application and server. This module is a creation of pages for each interaction of application as backup to the server and restore from the server.

Write the query in sql format for taking backup from device and another query for restoration from the server i.e. my sql database.Now this is to be done for every task that is we define in application.

For example, contact backup query

\$query="INSERT INTO

sharedcontacts(userid,dob,name,number,type)VALUES(". \$userid.",'".\$dob."',"'.\$name."',"'.\$number."',".\$type."');"; mysqlStart('localhost','backup');

\section{Development of my sql}

Now for configure of my sql and php we need to install a server software i.e. wamp server.

This server having each field for specifying the attribute which required for interaction between device to php page and to my sql database. In this myphp admin is where we have defined our tables for application.

There is table for :

1.

2.

CONTACT

3. MESSAGE

4. CALENDER EVENT

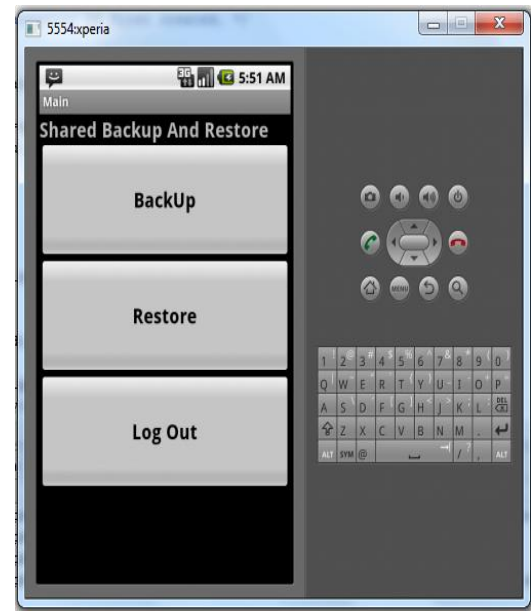

Fig 3: Application window

Each of the above table is specified with field with type of data to be store in table.

\begin{tabular}{|c|c|c|c|c|c|c|c|}
\hline \multicolumn{3}{|c|}{$\leftarrow \top \rightarrow$} & id & username & loginpassword & contact & email \\
\hline$\square$ & 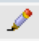 & $x$ & 4 & sangharsh & sangharsh & 9822334455 & sangharsh@gmail.com \\
\hline 口 & $\theta$ & $x$ & 5 & rahul & rahul & 998776876 & kjghlkjg@kjgk.com \\
\hline$\square$ & 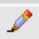 & $x$ & 6 & dinesh & dinesh & 43464646 & dinesh@gmail.com \\
\hline$\square$ & $\theta$ & $x$ & 7 & taher & taher & 765484825487 & taher@gmail.com \\
\hline
\end{tabular}

Fig 4: User login table

\section{Backup module}

For this module we need create login for accessing application then only we can create backup. This module display the list of application from which application we need backup (call logs, contacts, message.). This only the using the created work for backup for taking backup of device by the help of php page and mysql database table which is now communicate through the application. The backup is take from the device by date wise so later on we can take restore by choosing appropriate date.

Now for contact restore query

\$query="SELECT * FROM contacts;";

mysq1Start('localhost','backup'); 
IOSR Journal of Engineering

Mar. 2012, Vol. 2(3) pp: 433-436

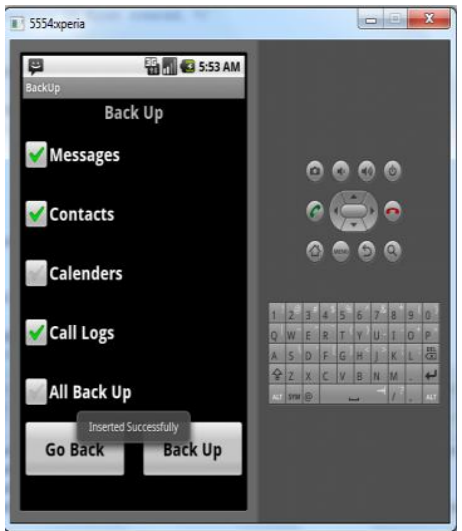

Fig 5: selecting backup

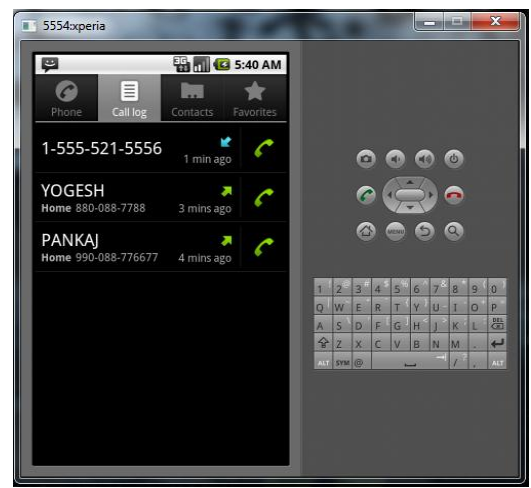

Fig 6: Call logs on Device

\begin{tabular}{|c|c|c|c|c|c|c|c|c|}
\hline$\vdash \top \nrightarrow$ & id use & serid & dob & name & number & type & duration & date \\
\hline $0 x$ & 1 & 4 & 18012012 & sangharsh & 88778 & 2 & 17 & 13269 \\
\hline $0 x$ & 2 & 4 & 18012012 & sangharsh & 887788887777 & 2 & 39 & 1326904495183 \\
\hline $0 x$ & 3 & 7 & 20012012 & PANKAJ & 99000 & 2 & 45 & 554821 \\
\hline $0 x$ & 4 & 7 & 20012012 & YOGESH & 8800887788 & 2 & 36 & 13270 \\
\hline $0 x$ & 5 & & 20012012 & & 15555215556 & 1 & 62 & \\
\hline
\end{tabular}

Fig 7: After taking Backup

\section{Restore module}

For this module we need access application using created login username and password.

This module display content list for which application we need restoration (call logs, contacts, messages..). Again using the created operation in php and mysql we can easily restore data from database to device.

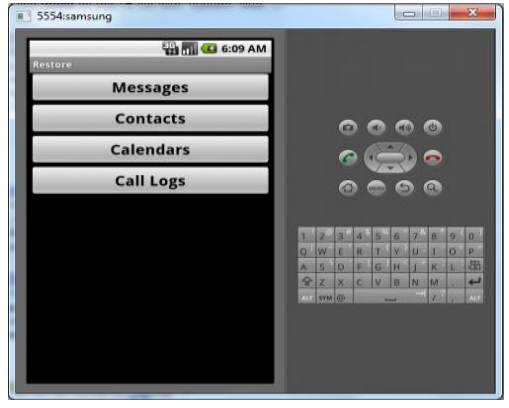

Fig 8: Restore window

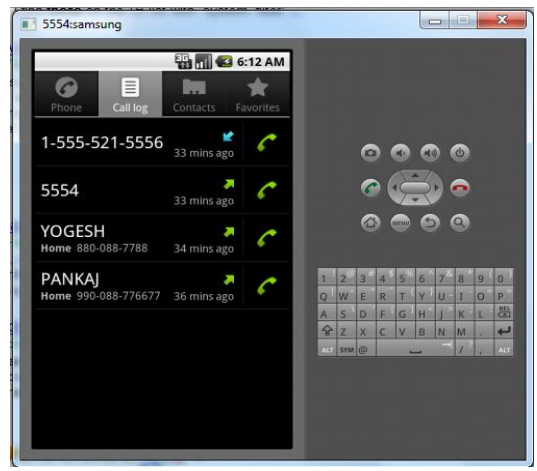

Fig 9: After Restoration on new device

\section{CONCLUSION}

The presented work here can be enriched with many refinement which are now in study phase.It 's however represents a highly encouraging first approach of defining system of backup and restore on on-line server in minimize time.

\section{REFERNCES}

[1] A.Chervenak, V. Vellanki, and Z.kurmas, Protecting File Systems: A Survey of Backup Techniques. Proceeding Joint NASA and IEEE Mass Storage 1998.

[2] BELL, T. C., CLEARY, J. G., AND WITTEN, I. H. Text Compression. Prentice Hall, Upper Sadle River, NJ, 1990.

[3] SAYOOD, K. Introduction to Data Compression. Academic Press, San Diego, CA, 1996, 2000.

[4] http://developer.android.com/guide/topics/data/backup.ht $\mathrm{ml}$.

[5] http://developer.android.com/guide/topics/providers/conte nt-providers.html

[6] http://en.wikipedia.org/wiki/ZIP_\%28file_format\%29. 